\title{
MRI Spectrum of Japanese Encephalitis in Northeast India: A Cross-Sectional Study
}

\author{
Pranjal Phukan ${ }^{1}$ Kalyan Sarma ${ }^{2}$ Barun Kumar Sharma ${ }^{3}$ Deb K. Boruah ${ }^{4}$ Bidyut Bikash Gogoi ${ }^{5}$ \\ Daniala Chuunthang ${ }^{1}$
}

${ }^{1}$ Department of Radiodiagnosis, North Eastern Indira Gandhi Regional Institute of Health and Medical Sciences, Shillong, Meghalaya, India

2Department of Neuroradiology, All India Institute of Medical Sciences, New Delhi, India

${ }^{3}$ Department of Radiodiagnosis, Sikkim Manipal Institute of Medical Sciences, Gangtok, Sikkim, India

${ }^{4}$ Department of Radiodiagnosis, Tezpur Medical College, Sonitpur, Assam, India

${ }^{5}$ Department of Pathology, Assam Medical College, Dibrugarh,

Assam, India

\begin{abstract}
Address for correspondence Deb K. Boruah, MD, PDCC, Department of Radiodiagnosis, MRI Room, Tezpur Medical College, Sonitpur, Tezpur 784010, Assam, India (e-mail: drdeb_rad@yahoo.co.in).
\end{abstract}

J Neurosci Rural Pract 2021;12:281-289.
Abstract
Keywords
- magnetic resonance imaging
- acute encephalitic syndrome
- encephalitis
- diffusion-weighted imaging
- autoimmune disease

Objective Japanese encephalitis (JE) is an arthropod-borne flavivirus infection having high mortality and morbidity. This study was performed to evaluate the conventional magnetic resonance imaging (MRI) findings in JE and to find out any difference between pediatric and adult JE.

Materials and Methods This retrospective study was performed on serologically positive $54 \mathrm{JE}$ patients presented to a tertiary care hospital with acute encephalitic symptoms between April 2016 and October 2019. Relevant neurological examination, cerebrospinal fluid analysis, and MRI scan of the brain were performed.

Results Fifty-four JE patients ( $n=31$ males and $n=23$ females) having 32 pediatric and 22 adult JE were included in the study sample. Group $1 \mathrm{JE}(n=16)$ patients had encephalitic symptoms with duration less than 15 days up to the day of MRI scan and group $2 \mathrm{JE}(n=38)$ had symptoms more than 15 days. Group 1 JE had mean apparent diffusion coefficient (ADC) value of $0.563 \pm 0.109$ (standard deviation [SD]) $\times$ $10^{-3} \mathrm{~mm}^{2} / \mathrm{sec}$ and group $2 \mathrm{JE}$ had $1.095 \pm 0.206(\mathrm{SD}) \times 10^{-3} \mathrm{~mm}^{2} / \mathrm{sec}$. The mean ADC value of pediatric JE was $0.907 \pm 0.336(\mathrm{SD}) \times 10^{-3} \mathrm{~mm}^{2} / \mathrm{sec}$ and adult JE was $0.982 \pm$ $0.253(\mathrm{SD}) \times 10^{-3} \mathrm{~mm}^{2} / \mathrm{sec}$.

Conclusion The majority of the JE patient shows abnormal signal alterations in bilateral thalami and substantia nigra. Diffusion-weighted imaging with ADC mapping helps in evaluating the stage of the JE. No statistical significance of the various conventional MRI findings was found between the pediatric JE and adult JE. published online

March 24, 2021
DOI https://doi.org/

$10.1055 / \mathrm{s}-0041-1722820$

ISSN 0976-3147. (c) 2021. Association for Helping Neurosurgical Sick People.

This is an open access article published by Thieme under the terms of the Creative Commons Attribution-NonDerivative-NonCommercial-License, permitting copying and reproduction so long as the original work is given appropriate credit. Contents may not be used for commercial purposes, or adapted, remixed, transformed or built upon. (https://creativecommons.org/licenses/by-nc-nd/4.0/).

Thieme Medical and Scientific Publishers Pvt. Ltd. A-12, 2nd Floor, Sector 2, Noida-201301 UP, India 


\section{Introduction}

Japanese B encephalitis (JE) is one of the mosquito-borne flaviviral infections that is transmitted through mosquito bites. JE is one of the common causes of acute encephalitis syndrome (AES). JE is a common health problem in countries like Japan, Korea, and India. ${ }^{1}$ Recurrent outbreak of JE are reported annually, between the month of July and October from the districts of upper Assam of Northeast India. ${ }^{2} \mathrm{JE}$ had an estimated annual incidence of 30,000 to 50,000 and had 15,000 deaths per year in children below 15 years in India. . $^{3-5}$

In the endemic area, children below 15 years of age are more vulnerable and even more prone in 3 to 6 years of age. ${ }^{5}$

The majority of the infection is asymptomatic, with only 20 to $30 \%$ of patients presenting with overt symptoms or fatality. ${ }^{6}$ About 30 to $50 \%$ of survivors of acute severe JE had long-term abnormal neurological, behavioral, cognitive sequelae, and extrapyramidal syndrome, especially in children. ${ }^{3,6}$ The case fatality rate of acute severe symptomatic JE patients increases up to $30 \%{ }^{6}$

The JE lesions are commonly observed in thalami, substantia nigra, and basal ganglia with less involvement of brain stem, cerebral cortices, cerebellum, cerebral white matters, and spinal cord. ${ }^{4,7}$ However, the distribution, extent, number of involved sites, and sizes of these lesions might vary in the pediatric JE and adult JE.

Moreover, T2 weighted image (T2WI) hyperintensities in thalami have high specificity for JE in the endemic area. ${ }^{4.8}$ The most common differentials of thalamic T2WI hyperintensities include hypoxic-ischemic encephalopathy, Wernicke's encephalopathy, herpes encephalitis, Wilson's disease, deep vein thrombosis, artery of Percheron infarct, Creutzfeldt-Jakob disease (CJD), and other flavivirus encephalitis, such as, dengue encephalitis and West Nile encephalitis. ${ }^{9}$

Atypically, JE with temporal lobe dominance can be difficult to differentiate from herpes encephalitis and autoimmune encephalitis. ${ }^{10}$ Autoimmune encephalitis most commonly affects bilateral mesial temporal lobes and limbic systems with less involvement of lateral temporal lobe and insular cortices with typical presentation of gradual short-term memory loss and mental status changes without acute encephalitic symptoms. ${ }^{11}$ Typical involvement of medial temporal lobes, insular cortex, and orbitofrontal lobes was observed in herpes simplex virus 1 . The posterior medial thalamus is commonly involved in JE whereas the anterior lateral thalamus is involved in Wilson disease. ${ }^{12} \mathrm{JE}$ can also cause deep venous sinus thrombosis in children. ${ }^{13,14}$ Furthermore, the bilateral thalamic T2W hyperintensities in deep vein thrombosis is usually associated with hemorrhages. ${ }^{15}$ The typical magnetic resonance imaging (MRI) findings of CJD show symmetrical bilateral posterior thalamic T2W hyperintensities giving "pulvinar sign" and dorsomedial thalamic nuclei hyperintensities giving "hockey stick sign." ${ }^{16,17}$ Also, lack of fever and other acute encephalic symptoms in CJD helps to differentiate it from acute JE. ${ }^{16,17}$

The distribution, extension, number of involved sites, and sizes of these lesions might vary in pediatric and adult JE. These differences in MRI abnormalities in pediatric and adult JE might help in neuroradiological diagnosis, and possibly have implications on treatment plans.

This study was performed to evaluate the conventional MRI findings in JE and to find out any difference between pediatric and adult JE.

\section{Materials and Methods}

A retrospective cross-sectional study was conducted at our institution on serologically positive $54 \mathrm{JE}$ patients presented with acute encephalitic symptoms between April 2016 and October 2019. This retrospective study was approved by the institutional ethics review committee.

\section{MRI Protocols}

The patients underwent an MRI scan of the brain, using a 1.5T MR scanner, Siemens Magnetom Avanto (Siemens Medical Systems, Erlangen, Germany). Conventional MRI sequences protocol includes axial T1WI, T2WI, fluid-attenuated inversion recovery (FLAIR), diffusion-weighted imaging (DWI), and susceptibility-weighted imaging (SWI) sequences followed by sagittal T1WI and coronal T2WI sequences. Post-gadolinium T1WI sequences were obtained in all three planes. The parameters of the various MRI sequences used are shown in - Table 1.

Table 1 Showing parameters used in various conventional MRI sequences

\begin{tabular}{|l|l|l|l|l|l|l|l|}
\hline MRI sequence & TE $(\mathrm{ms})$ & TR $(\mathrm{ms})$ & Matrix & $\begin{array}{l}\text { Field of view } \\
(\text { FOV) }\end{array}$ & $\begin{array}{l}\text { Slice thickness } \\
(\mathrm{mm})\end{array}$ & $\begin{array}{l}\text { Flip } \\
\text { angle }\end{array}$ & Others \\
\hline T2W axial & $90-110$ & $3,800-6,000$ & 512 & $220-250$ & 5 & $150^{\circ}$ & $150^{\circ}$ \\
\hline T1W axial & $8-10$ & $500-600$ & 512 & $220-250$ & 5 & $150^{\circ}$ & $\mathrm{TI}=2,500 \mathrm{~ms}$ \\
\hline FLAIR axial & $90-100$ & 9,000 & 512 & $220-250$ & 5 & $90^{\circ}$ & $\begin{array}{l}b-\mathrm{value}=0 \text { and } \\
1,000 \mathrm{sec} / \mathrm{mm}^{2}\end{array}$ \\
\hline DWI axial & $90-110$ & $3,000-4,000$ & 128 & $220-250$ & 5 & $15^{\circ}$ & \\
\hline SWI axial & 40 & $50-60$ & 256 & $220-250$ & 2 & $90^{\circ}$ & \\
\hline T1W sagittal & $8-10$ & $500-600$ & 256 & $220-250$ & 4 & $150^{\circ}$ & \\
\hline T2W coronal & $80-95$ & $4,000-6,000$ & 512 & $220-250$ & 4 & \\
\hline
\end{tabular}

Abbreviations: DWI, diffusion-weighted imaging; FLAIR, fluid-attenuated inversion recovery; MRI, magnetic resonance imaging; SWI, susceptibility weighted imaging; TE, time of echo; TI, time of inversion; TR, repetition time. 


\section{Serological Test}

Confirmation of JE was established by immunoglobulin $\mathrm{M}$ antibodies positivity to the JE virus in cerebrospinal fluid and serum samples by enzyme-linked immunosorbent assay technique.

\section{Apparent Diffusion Coefficient Calculation}

The apparent diffusion coefficient (ADC) values were measured independently by two radiologists and the results were obtained from the mean ADC values. Calculation of the mean ADC values was done by placing either round or elliptical regions of interest (ROIs). We measured the mean $A D C$ value in the operating system console using three uniform size ROIs (with area: maximum $50 \mathrm{~mm}^{2}$ and minimum $10 \mathrm{~mm}^{2}$ ) placed in the ADC map image of $b=1,000 \mathrm{sec} / \mathrm{mm}^{2}$, and the mean $A D C$ value was calculated for statistical analysis.

\section{Statistical Analysis}

All statistical analysis was performed using Statistical Package for Social Science (SPSS, version 16). A nonparametric Wilcoxon rank test was performed to find out the difference between group 1 and group 2 of JE patients. An independent sample Student's $t$-test was used to compare the mean ADC values between pediatric and adult JE patients. Statistical significance was considered when the $p$-value was $\leq 0.05$.

\section{Results}

Of the $54 \mathrm{JE}$ patients of our study, 31 were males and 23 were females with a mean age of $22.9 \pm 1.9$ (standard deviation [SD) years. The age range was 7 months to 73 years. Patients aged less than 18 years were categorized into pediatric JE $(n=32)$ group and those above 18 years into adult JE $(n=22)$ group. JE patients with duration of onset of acute encephalitic symptoms like high fever, headache, neck stiffness, and altered mental status less than 15 days up to the day of MRI scan were categorized into group $1 \mathrm{JE}(n=16)$ and those more than 15 days into group $2 \mathrm{JE}(n=38)$. The mean day of MRI examination from the time of onset of acute encephalitic symptoms was $14.3 \pm 4.6$ (SD). Group $1 \mathrm{JE}$ comprised of 16 patients (29.6\%) and group 2 JE comprised of 38 patients $(73.4 \%)$.

Diffusion restriction with low ADC value means the true diffusion restriction in acute JE patients. It indicates that group $1 \mathrm{JE}$ patients ( $\mathbf{- F i g s .} \mathbf{1}$ and $\mathbf{2}$ )were imaged at the stage of perivascular cuffing leading to ischemic and cytotoxic edema, which resulted in true diffusion restriction. Statistical significance was found between the duration of symptoms onset up to the day of MRI examination and mean ADC values of JE lesions with $p$-value $<0.0005$ by using nonparametric two related samples Wilcoxon signed rank test. The various brain MRI findings of JE are summarized in - Table 2.
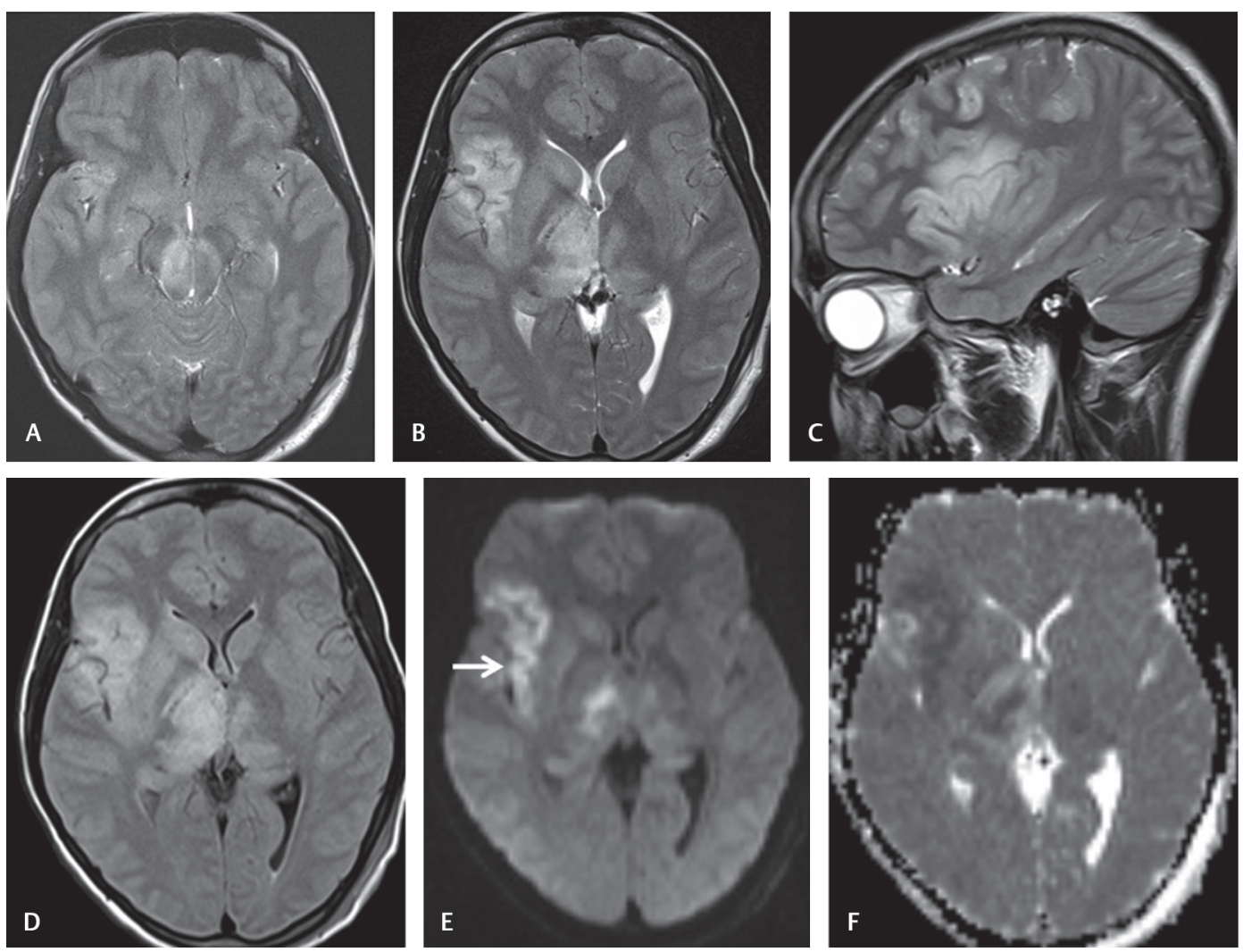

Fig. 1 Cranial magnetic resonance imaging was done on day 9 on 28 female patients with acute encephalitic symptoms. Axial T2WI (A and B) and sagittal T2WI (C) images showing asymmetrical hyperintensities in bilateral substantia nigra and thalami with the affection of the right insular cortex. Axial fluid-attenuated inversion recovery image (D) also showing the abnormalities. Axial diffusion-weighted imaging (E) and apparent diffusion coefficient (ADC) map (F) images showing diffusion restrictions in the affected regions with low ADC value (arrow). 

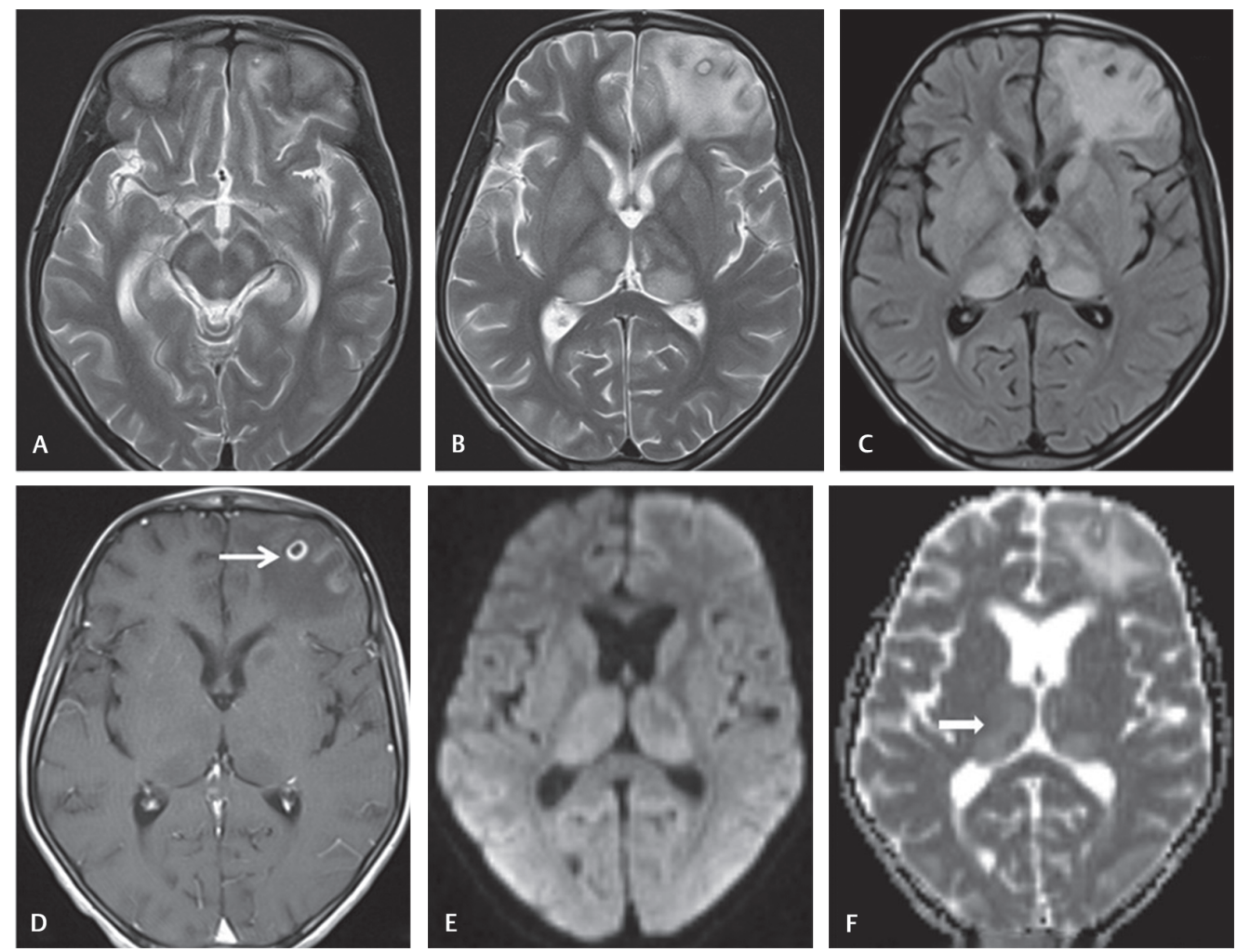

Fig. 2 Cranial magnetic resonance imaging was done on day18 on an 11 years old female with fever and headache. Axial T2WI (A and B) and axial fluid-attenuated inversion recovery $(\mathbf{C})$ images showing hyperintensities in bilateral thalami, substantia nigra, and left caudate head along with a central T2 hyperintense and peripheral hypointense cyst in the left frontal lobe with perifocal edema. Axial T1W postcontrast image (D) showing rim enhancement of the left frontal lobe neurocysticercosis lesion (arrow). Axial diffusion-weighted imaging (E) and apparent diffusion coefficient map (F) images showing facilitated diffusion in the thalami (black arrow).
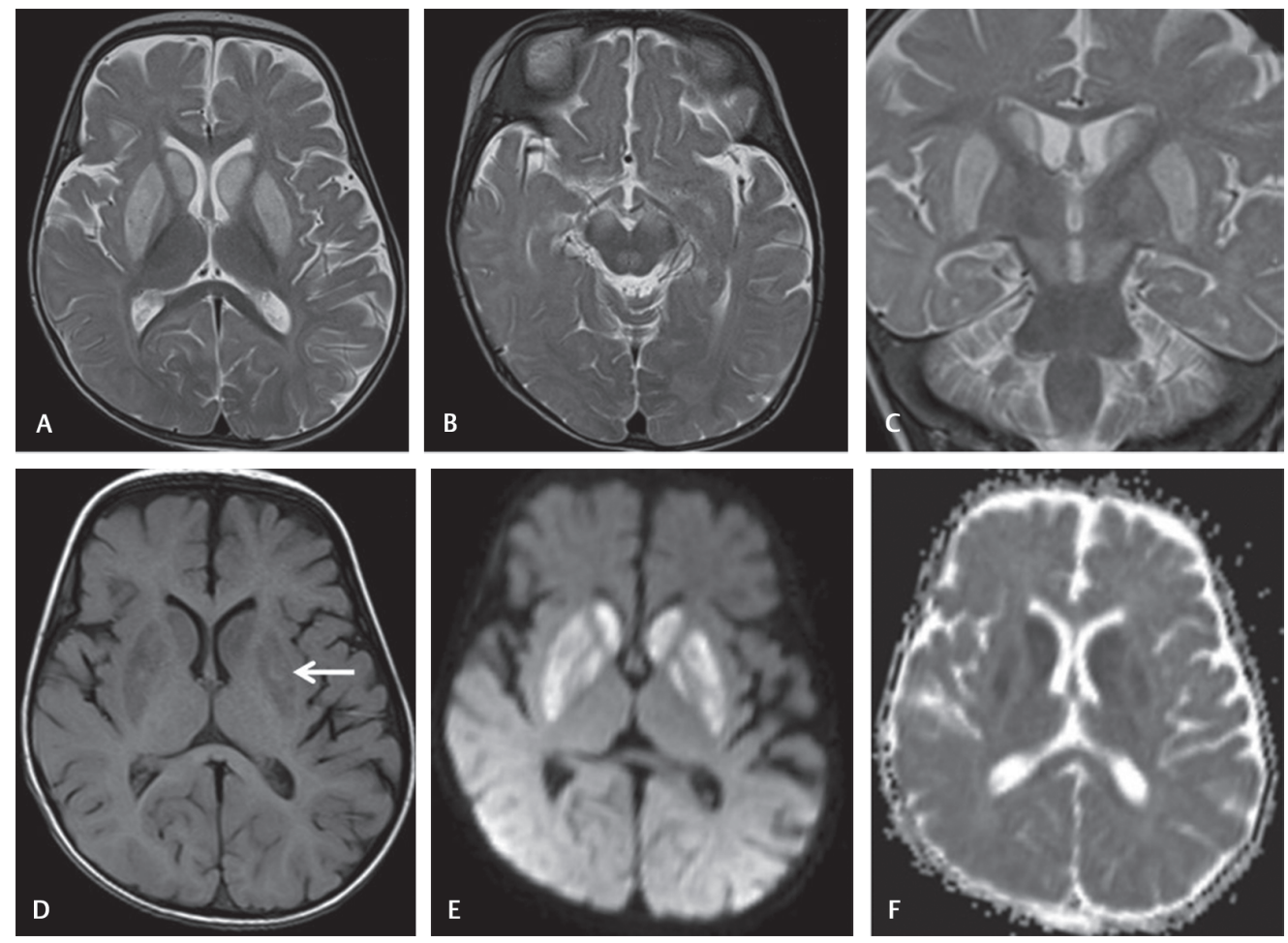

Fig. 3 Cranial magnetic resonance imaging was done on day 6 on a 7-month-old male baby with acute encephalitic symptoms. Axial T2WI (A and $\mathbf{B}$ ) and coronal T2WI (C) images showing hyperintensities in bilateral putamen, caudate nuclei, and substantia nigra without involvement of thalami. Axial T1WI image (D) showing patchy T1 hyperintense bleeds in the bilateral putamen. Axial diffusion-weighted imaging (E) and apparent diffusion coefficient (ADC) map (F) images showing diffusion restrictions with low ADC value. 


\begin{tabular}{|c|c|c|c|c|c|c|c|c|c|c|c|c|c|c|c|c|c|c|c|c|c|c|c|c|c|c|c|c|}
\hline \multirow{3}{*}{ 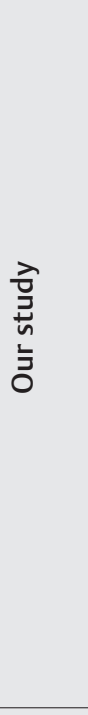 } & $\begin{array}{l}\frac{\omega}{5} \approx \\
\frac{5}{2} \\
\frac{2}{2}\end{array}$ & 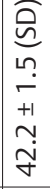 & 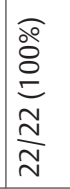 & 1 & $\begin{array}{l}\text { ㅇ } \\
\frac{0}{2} \\
\frac{N}{N}\end{array}$ & 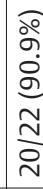 & 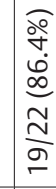 & & 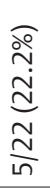 & 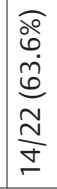 & 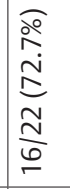 & 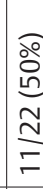 & 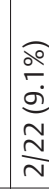 & 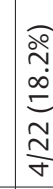 & 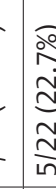 & 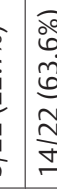 & 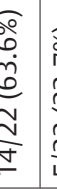 & 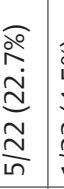 & 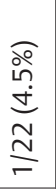 & 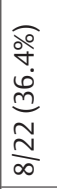 & 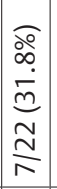 & 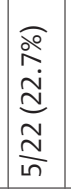 & 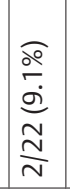 & 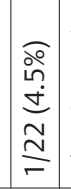 & 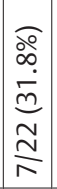 & 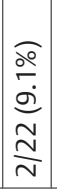 & 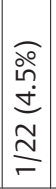 & 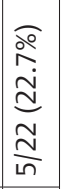 \\
\hline & 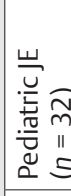 & 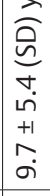 & 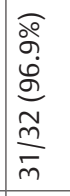 & 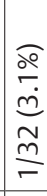 & 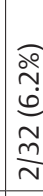 & 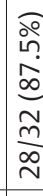 & 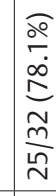 & & 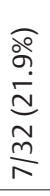 & 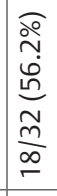 & 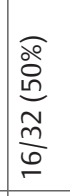 & 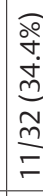 & 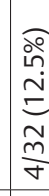 & 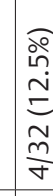 & $\left\{\begin{array}{l}\partial \\
\stackrel{o}{\sigma} \\
\frac{m}{m} \\
\frac{m}{m}\end{array}\right.$ & 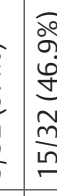 & 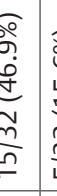 & 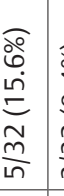 & 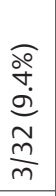 & 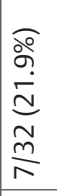 & 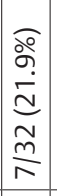 & 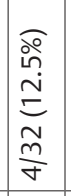 & $\begin{array}{l}\widehat{o} \\
\dot{\sigma} \\
\stackrel{\sigma}{\sigma} \\
\tilde{m} \\
\frac{m}{m}\end{array}$ & 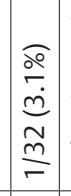 & 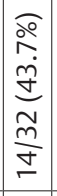 & $\begin{array}{l}\frac{\widehat{o}}{\stackrel{\rho}{\sigma}} \\
\stackrel{\sigma}{\tilde{m}} \\
\frac{\tilde{m}}{m}\end{array}$ & 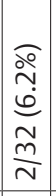 & 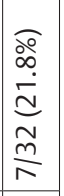 \\
\hline & 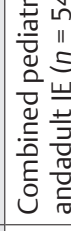 & 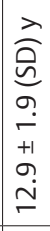 & 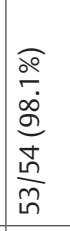 & 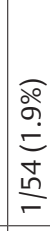 & 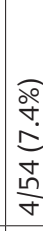 & $\begin{array}{l}\text { ळे } \\
\infty \\
\infty \\
\infty \\
5 \\
\frac{1}{\circ} \\
\stackrel{\infty}{+}\end{array}$ & 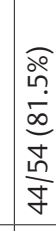 & & 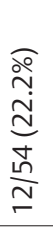 & 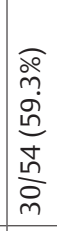 & 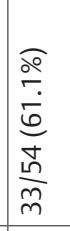 & 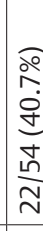 & 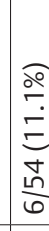 & 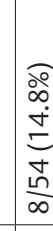 & 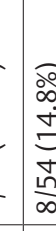 & 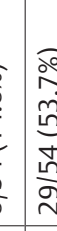 & 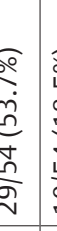 & 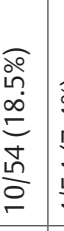 & 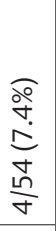 & 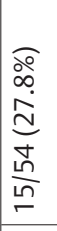 & 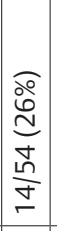 & 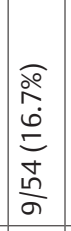 & 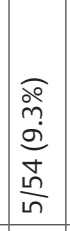 & 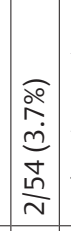 & 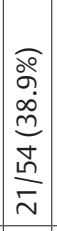 & 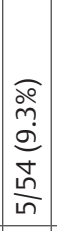 & 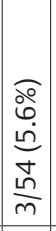 & 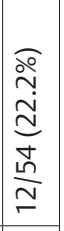 \\
\hline 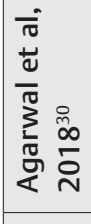 & 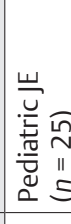 & 1 & 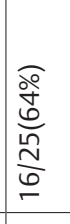 & 1 & I & 1 & 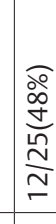 & & 1 & 1 & 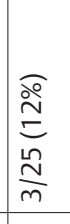 & 1 & & & & & 1 & & & & 1 & & & & 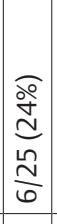 & 1 & 1 & 1 \\
\hline 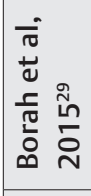 & 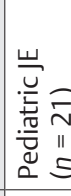 & 1 & 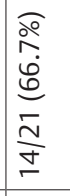 & $\begin{array}{l}\stackrel{\circ}{\check{~}} \\
\stackrel{m}{0}\end{array}$ & $\begin{array}{l}\stackrel{\circ}{m} \\
m \\
m\end{array}$ & $\begin{array}{l}\grave{0} \\
\infty \\
i\end{array}$ & 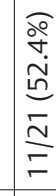 & & & & 1 & 1 & I & I & & & 1 & 1 & 1 & 1 & 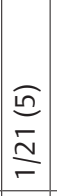 & & & 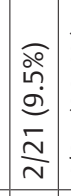 & 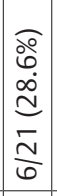 & & 1 & 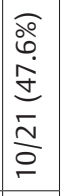 \\
\hline \multirow{2}{*}{ 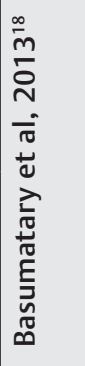 } & 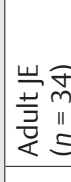 & 1 & 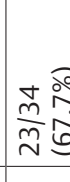 & & & & $\frac{\vec{m}}{r}$ & jo & & & 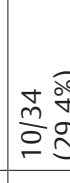 & & & & & & & & & & 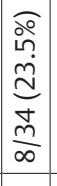 & & & 1 & 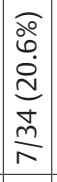 & 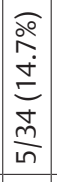 & $\begin{array}{l}1 \\
1 \\
\end{array}$ & 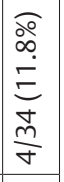 \\
\hline & 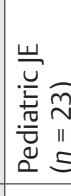 & 1 & 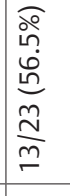 & 1 & 1 & I & 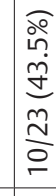 & & 1 & 1 & 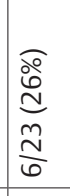 & & & & & & & & & & 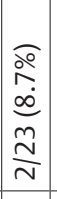 & 1 & & 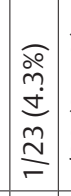 & 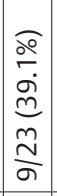 & 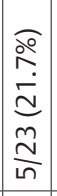 & 1 & 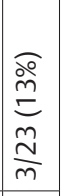 \\
\hline \multirow{2}{*}{ 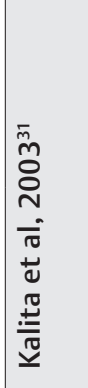 } & 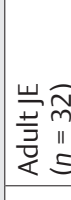 & & 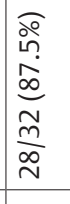 & & & & $\begin{array}{l}\widehat{o} \\
\stackrel{0}{\circ} \\
\stackrel{0}{d} \\
\tilde{N} \\
\frac{m}{\sigma}\end{array}$ & & & & 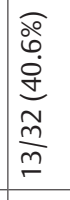 & & & & & & & & & & 1 & & & 1 & 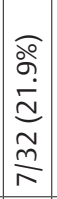 & 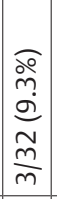 & I & \\
\hline & 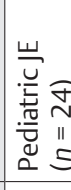 & 1 & 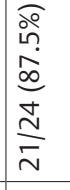 & 1 & 1 & 1 & 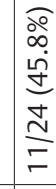 & & 1 & 1 & 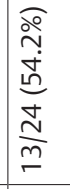 & 1 & & & & & 1 & & & & 1 & & & & 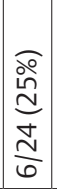 & $\mid \begin{array}{c}\stackrel{o}{o} \\
m \\
\infty \\
\stackrel{d}{d} \\
\frac{N}{N}\end{array}$ & . & 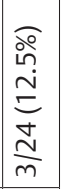 \\
\hline 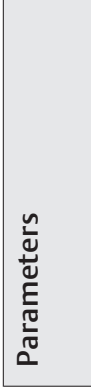 & 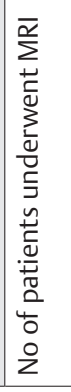 & 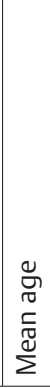 & 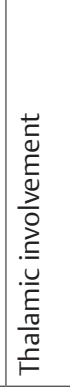 & 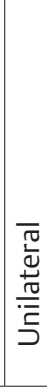 & 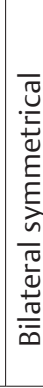 & 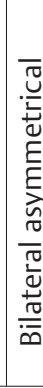 & 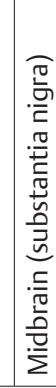 & 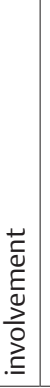 & 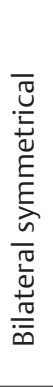 & 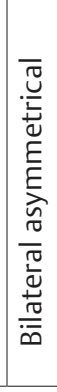 & 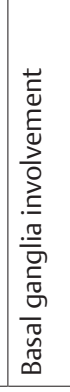 & 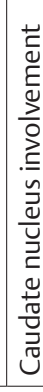 & 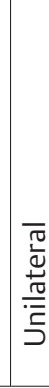 & 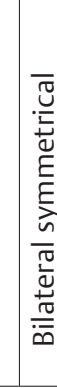 & 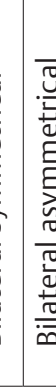 & 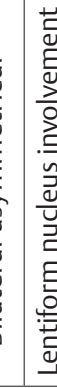 & 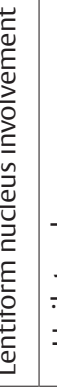 & . & 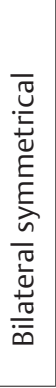 & 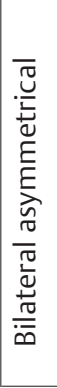 & 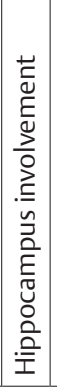 & 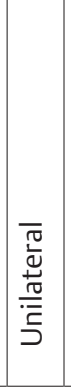 & 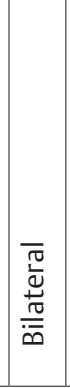 & 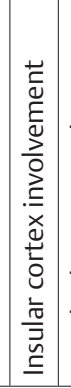 & 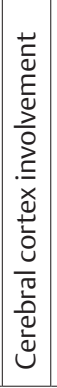 & 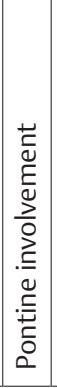 & 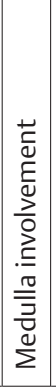 & 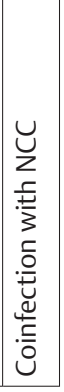 \\
\hline
\end{tabular}



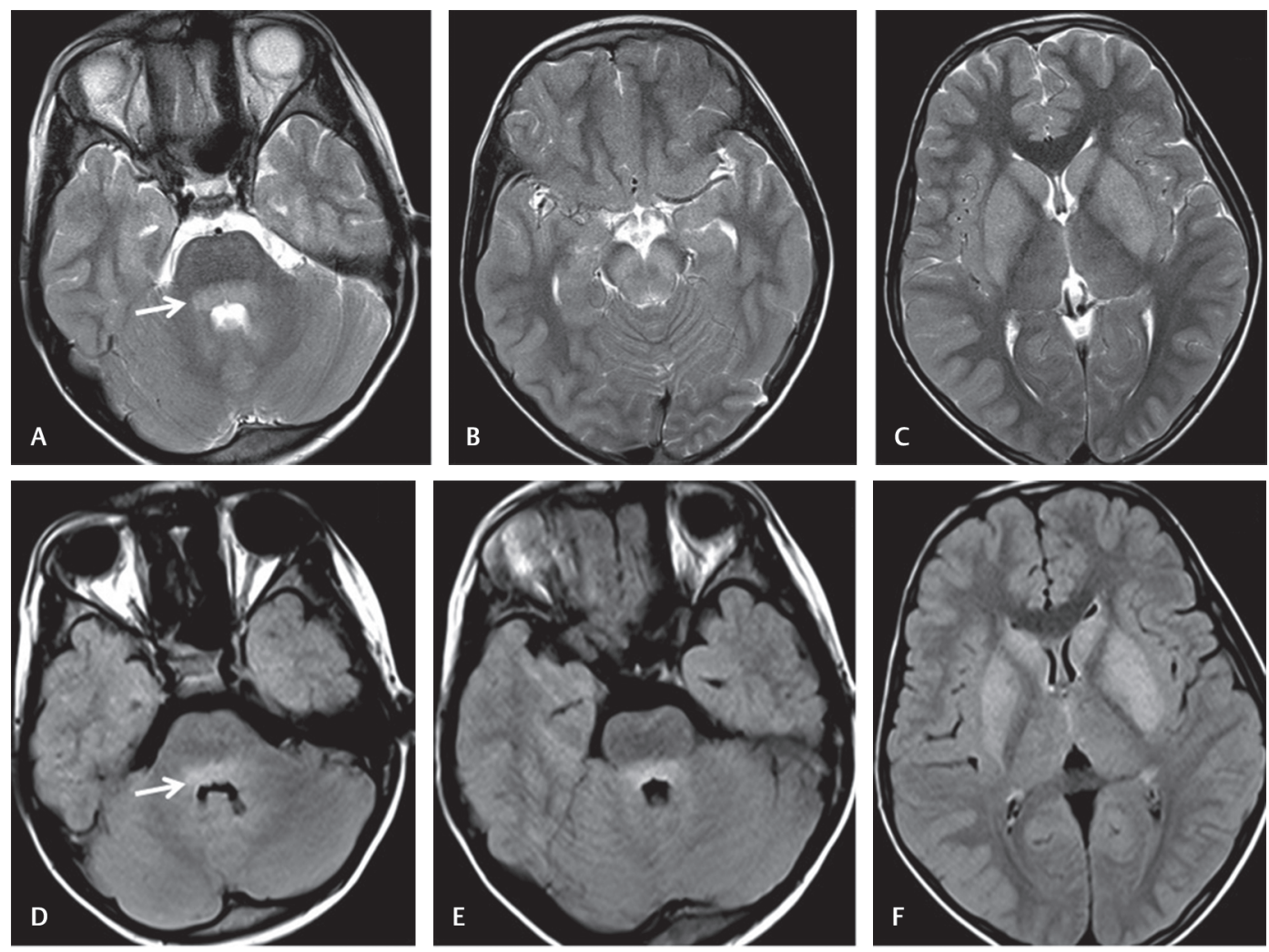

Fig. 4 Cranial magnetic resonance imaging was done on day 5 on a 9 years old male with headache and fever. Axial T2WI images (A-C) showing hyperintensities in basilar pons, bilateral substantia nigra, caudate, and lentiform nuclei. Axial fluid-attenuated inversion recovery images (D-F) showing hyperintensities in the basilar portion of pons (arrow), bilateral caudate, and lentiform nuclei.

Pediatric JE patients had mean ADC value of $0.907 \pm 0.336$ $(\mathrm{SD}) \times 10^{-3} \mathrm{~mm}^{2} / \mathrm{s}$ and adult JE had $0.982 \pm 0.253(\mathrm{SD}) \times$ $10^{-3} \mathrm{~mm}^{2} / \mathrm{s}$. No difference was found between the mean ADC value of pediatric JE and adult JE patients by using an independent sample Student's $t$-test ( $p$-value of 0.388 ). Group $1 \mathrm{JE}$ had a mean ADC value of $0.563 \pm 0.109(\mathrm{SD}) \times 10^{-3} \mathrm{~mm}^{2} / \mathrm{s}$ and group $2 \mathrm{JE}$ had $0.095 \pm 0.206(\mathrm{SD}) \times 10^{-3} \mathrm{~mm}^{2} / \mathrm{s}$ with a significant difference between these two groups by using the Wilcoxon signed-rank test ( $p$-value $<0.0005$ ).

T2WI and FLAIR thalamic hyperintensities were demonstrated in 53 patients (98.1\%), bilaterally asymmetrical in 48 patients (88.9\%) (-Figs. 1 and 2 ), bilaterally symmetrical in 4 patients (7.4\%), and unilateral in another 1 patient (1.9\%). Bilateral substantia nigra involvement was observed in 44 patients (81.5\%), bilaterally asymmetrical in 30 patients (59.3\%) (-Fig. 1), and bilaterally symmetrical in 12 patients (22.2\%) (-Fig. 2). Hippocampal involvement was observed in 14 patients (26\%), unilateral in 9 patients (16.7\%), and bilateral in 5 patients (9.3\%). Total hippocampal involvement was observed in 4 patients (7.4\%), hippocampal head and body involvement in 7 patients (13\%), and only tail involvement in 3 patients (5.6\%). Caudate nuclei involvement was observed in 22 patients (40.7\%), bilaterally symmetrical in 8 patients (14.8\%) (-Fig. 3), bilaterally asymmetrical in 8patients (14.8\%), and unilateral in 6 patients (11.1\%) ( - Fig. 2). Lentiform nuclei involvement was observed in 29 patients (53.7\%), bilaterally asymmetrical in 15 patients (27.8\%), bilaterally symmetrical in 4 patients (7.4\%) (-Figs. 3 and 4), and unilateral in 10 patients (18.5\%). Insular cortex involvement was observed in 2 patients $(3.8 \%)$ (-Fig. 1). Cerebral cortex involvement was observed in 21 patients (38.9\%), with single cerebral hemispheric involvement observed in 13 patients (24.1\%) (-Fig. 5) and bilateral cerebral hemispheric involvement in 8 patients (14.8\%).

Cerebral cortex involvement in more than one lobe of the cerebral hemisphere was observed in 9 patients (16.7\%) (-Fig. 5) with only frontal lobe cortical involvement observed in another 8 patients (14.8\%). Involvement of pons was observed in 5 patients (9.3\%) ( - Fig. 4) and medulla oblongata involvement in 3 patients (5.6\%). On SWI, thalamic microbleeds were observed in 2 JE patients (3.7\%).

JE coinfection with neurocysticercosis (NCC) was observed in 12 patients (22.2\%) ( - Fig. 2), with the involvement of one cerebral hemisphere in 6 patients $(11.1 \%)$ and bilateral cerebral hemispheres in another 6 patients (11.1\%).

\section{Discussion}

Abnormal T2 and FLAIR hyperintensities of typical JE may be observed in thalami, ${ }^{4,718,19}$ substantia nigra, basal ganglia, ${ }^{19-21}$ hippocampus, and pons and less commonly in the cortical and subcortical regions $s^{4,8,22}$ and cerebellar 

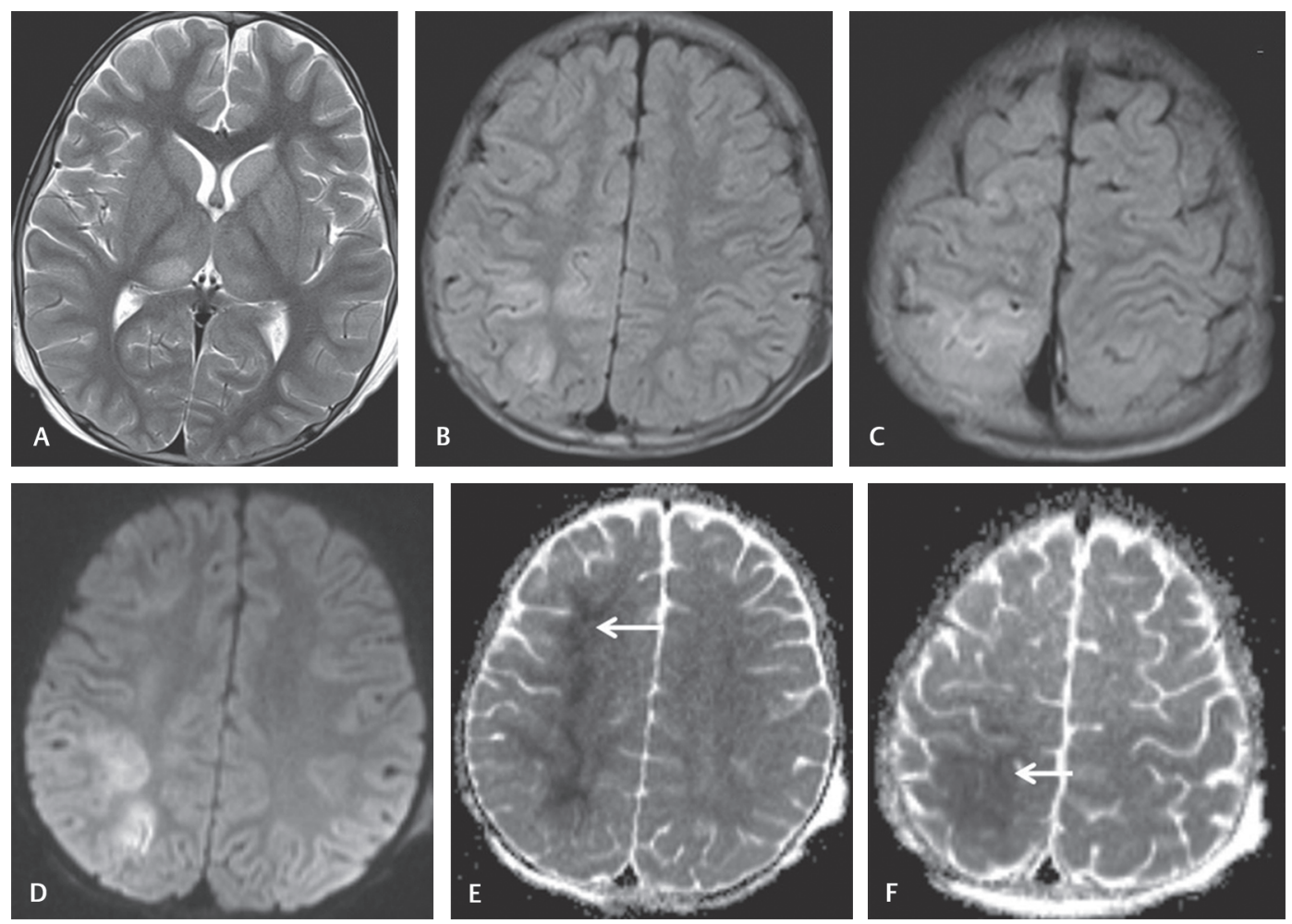

Fig. 5 Cranial magnetic resonance imaging was done on day 7 on a 5 -year-old boy with acute encephalitic symptoms. Axial T2WI (A) image showing hyperintensities in the bilateral thalami. Axial fluid-attenuated inversion recovery (FLAIR) images (B and C) showing hyperintensities in the cortical and subcortical regions of right superior frontoparietal lobes. Axial diffusion-weighted imaging (D) showing diffusion restrictions in the affected cortices. Apparent diffusion coefficient (ADC) map ( $\mathbf{E}$ and $\mathbf{F}$ ) images showing pronounced hypointense signals of low ADC value in the subcortical white matters of right superior frontoparietal lobes and overlying cortices (arrow).

hemisphere. The conventional MRI sequences like T2WI, FLAIR, and DWI can detect most of the abnormalities in JE patients. ${ }^{22}$ However, in newborns and infants, DWI can detect acute encephalitic lesions, much earlier than conventional T2WI and FLAIR sequences. ${ }^{23}$ This is because of more water content in the immature brain leading to problems with achieving conspicuity between the normal brain and encephalitic lesions in T2WI and FLAIR images. ${ }^{23}$ DWI can detect cytotoxic edema which is the earliest sign of any encephalitic process. Thus, DWI is more sensitive in early cases of JE but less sensitive in late cases of JE. ${ }^{23}$ Consequently, DWI can detect brain parenchymal changes in JE within few hours or 1 day before any demonstrable changes are observed in T2WI and FLAIR images. ${ }^{23}$ Acute stage of JE shows diffusion restriction with low ADC value. ${ }^{24}$ ADC value gradually increases after the late acute stage and remains higher in the chronic stage. ${ }^{24}$

Arahata et $\mathrm{al}^{25}$ demonstrated the successive changes in DWI on MRI in the thalamus ranging from spotty appearance to uniform pattern and also emphasized that the JE virus may initially affect the unilateral thalamus with subsequent involvement of the other side.

Another study showed progressive T2 and FLAIR hyperintensities in the thalami in the early stage of the disease. ${ }^{26}$ With the subsequent progression of JE, the T2 shrine-through effect is observed in the lesions in the subacute stage, with gradually increasing $A D C$ value and fogging of bright signals on diffusion-weighted images. In the chronic stage, the affected areas showed gliosis, cavitations, and atrophy. ${ }^{25}$ The resolution of T2 and FLAIR hyperintensities usually occurred within 1 to 3 months of clinical recovery but may persist for a longer course, sometimes even up to 1 year. ${ }^{8} \mathrm{~A}$ repeat follow-up MRI, should usually be done after 1 to 3 months of clinical recovery of JE patient. ${ }^{8}$

MRI plays an important role in the radiological diagnosis of $\mathrm{JE}$, by detecting the distribution, extension, and status of the parenchymal lesions (whether acute, subacute, or chronic), and excluding other AES-induced encephalitis. Even though overlap imaging features of herpes encephalitis, autoimmune encephalitis, and temporal lobe dominant JE are there, but these can be differentiated by MRI by observing the simultaneously altered signal intensities in the thalami, substantia nigra, and hippocampus in JE patients. 7,10,27,28

In our study group, substantia nigra affection was commonly observed in $81.5 \%(44 / 54)$ patients as compared with the previous literature from India. ${ }^{18,29-31}$ - Table 2 shows the review literature on MRI findings of JE from India in the last decade. In our study group, medial temporal lobe involvement with dominant hippocampal affection was observed in $26 \%(14 / 54)$ patients. Handique et al ${ }^{28}$ found hippocampal involvement in 11 out of $62 \mathrm{JE}$ patients (17.7\%).

The characteristic temporal lobe involvement pattern which is seen in JE typically affects the hippocampus, 
Table 3 Summary of magnetic resonance imaging findings in common flavivirus encephalitis

\begin{tabular}{|c|c|c|c|}
\hline Type & Sites of affection & Cranial MRI findings & Associated findings \\
\hline Japanese encephalitis & $\begin{array}{l}\text { - Thalami and substantia nigra are } \\
\text { commonly involved } \\
\text { - Basal ganglia, pons, cerebral } \\
\text { cortex, and } \\
\text { cerebellum are sometime involved }\end{array}$ & $\begin{array}{l}\text { - T2 and FLAIR hyperintensities in } \\
\text { the affected areas with diffusion } \\
\text { restricted in early acute stage and } \\
\text { facilitated diffusion in subacute } \\
\text { stage } \\
\text { - Rarely hemorrhage can be seen } \\
\text { in JE }\end{array}$ & $\begin{array}{l}\text { - Neurocysticercosis coinfection } \\
\text { - Rarely dengue coinfection can } \\
\text { occur } \\
\text { - Rarely may associated with CVST }\end{array}$ \\
\hline Dengue encephalitis & $\begin{array}{l}\text { - Thalami and basal ganglia are } \\
\text { commonly affected } \\
\text { - Pons, medulla, cerebellum, cor- } \\
\text { pus callosum, and cerebral cortex } \\
\text { can be involved }\end{array}$ & $\begin{array}{l}\text { - T2 and FLAIR hyperintensities } \\
\text { with restricted diffusion } \\
\text { - Parenchymal or extra-axial bleed } \\
\text { can be seen and which is more } \\
\text { common in dengue encephalitis } \\
\text { than JE } \\
\text { - Diffuse cerebral edema may be } \\
\text { seen }\end{array}$ & $\begin{array}{l}\text { - Cerebellitis is more common } \\
\text { - Cortical laminar necrosis }\end{array}$ \\
\hline West Nile encephalitis & $\begin{array}{l}\text { - Brain stem, basal ganglia, and } \\
\text { corona radiata commonly affected } \\
\text { - Thalami, cerebral cortex, an } \\
\text { white matters may be involved }\end{array}$ & $\begin{array}{l}\text { - Isolated diffusion restrictions in } \\
\text { posterior limb of internal capsule/ } \\
\text { corona radiata } \\
\text { - T2 and FLAIR hyperintensities in } \\
\text { brain stem, basal ganglia, thalami, } \\
\text { etc. } \\
\text { - Meningitis changes seen }\end{array}$ & $\begin{array}{l}\text { - Enhancement of cauda equina } \\
\text { and lumbosacral nerve roots } \\
\text { - Spinal cord T2 hyperintensities } \\
\text { can be seen }\end{array}$ \\
\hline Tick-borne encephalitis & $\begin{array}{l}\text { - Cerebellum commonly affected } \\
\text { - Thalami, basal ganglia, and ante- } \\
\text { rior horn cell of spinal cord may be } \\
\text { affected }\end{array}$ & $\begin{array}{l}\text { - Leptomeningitis along cerebellar } \\
\text { folia and basal cisterns } \\
\text { - T2 and FLAIR hyperintensities in } \\
\text { thalami, basal ganglia, and } \\
\text { cerebellum }\end{array}$ & \\
\hline $\begin{array}{l}\text { Murray Valley } \\
\text { encephalitis } \\
\text { (Australian encephalitis) }\end{array}$ & $\begin{array}{l}\text { - Thalami and brain stem com- } \\
\text { monly affected }\end{array}$ & $\begin{array}{l}\text { - Bilateral symmetrical T2 and } \\
\text { FLAIR hyperintensities in thalami } \\
\text { and brain stem }\end{array}$ & \\
\hline St. Louis encephalitis & $\begin{array}{l}\text { - Substantia nigra most commonly } \\
\text { affected }\end{array}$ & $\begin{array}{l}\text { - T2 hyperintensities in substantia } \\
\text { nigra }\end{array}$ & -Myelitis can be seen rarely \\
\hline
\end{tabular}

Abbreviations: CVST, cerebral venous sinus thrombosis; FLAIR, fluid-attenuated inversion recovery; JE, Japanese encephalitis; MRI, magnetic resonance imaging.

especially its body and tail with sparing of the rest of the temporal lobe.

Previous literature showed a variable association of JE with NCC. ${ }^{32,33}$ Handique et a ${ }^{32}$ found JE with NCC coinfection in $19.3 \%$ (12/62) patients from Northeast India. In our study group, JE coinfection with NCC was found in $22.2 \%(12 / 54)$ patients. Moreover, five of these patients showed more confluent T2 hyperintensities in thalami, basal ganglia, and cerebral cortices in the NCC involved hemisphere. Furthermore, out of these 12 patients, poor clinical and neurological recovery was observed in 5 patients, with partial recovery in another 4 patients, and complete recovery in the rest of the 3 patients, on a follow-up period ranging from 12 to 18 months.

Sivamani et $\mathrm{al}^{34}$ reported JE coinfection with dengue virus infection in the same population as the transmission of both viruses intensifies during the rainy season. Dengue encephalitis most commonly showed abnormal T2 and FLAIR hyperintensities with restricted diffusions in thalami and basal ganglia. Less commonly pons, medulla, cerebellum, corpus callosum, and cerebral cortex can also be involved in dengue..$^{35}$ Intraparenchymal or extra-axial bleed is more common in dengue encephalitis than JE. Cerebellitis may also be associated with dengue encephalitis. ${ }^{35}$ - Table 3 summarizes the MR imaging findings in common flavivirus encephalitis.

JE with cerebral venous sinus thrombosis (CVST) is a rare occurrence with very few cases reports in the literature. ${ }^{36-38}$ In our study group, no CVST was detected.

Our study observed a higher incidence of multifocal lesions with greater involvement of cerebral cortices in pediatric JE compared with adult JE patients. However, no significant difference was observed between the cranial MRI findings in pediatric and adult JE patients ( - Table 2).

The lack of adequate statistical power in our observations to discriminate differences in MRI findings between pediatric and adult JE is most probably due to the small sample size. Nevertheless, these differences in the MRI findings between the pediatric and adult JE may be clinically significant in predicting the clinical outcome and neurological recovery, and might help in treatment planning. Therefore, a larger prospective study to confirm these findings is warranted in the future.

\section{Conclusion}

PediatricJE has a more severe form of encephalitis than adultJE with a poorer prognosis. No statistically significant difference 
in various brain MRI findings was observed between pediatric JE and adult JE. But a higher incidence of multifocal parenchymal lesions with greater involvement of cerebral cortices in pediatric JE as compared to adult JE was observed in our study group.

\section{Conflict of Interest}

None declared.

\section{References}

1 Monath TP. Japanese encephalitis-a plague of the Orient. N Engl J Med 1988;319(10):641-643

2 Dutta P, Khan SA, Khan AM, Borah J, Sarmah CK, Mahanta J. The effect of insecticide-treated mosquito nets (ITMNs) on Japanese encephalitis virus seroconversion in pigs and humans. Am J Trop Med Hyg 2011;84(3):466-472

3 Solomon T.Viral encephalitides in Southeast Asia. Neurol Infect Epidemiol. 1997;2:191-199

4 Kalita J, Misra UK. Comparison of CT scan and MRI findings in the diagnosis of Japanese encephalitis. J Neurol Sci 2000; $174(1): 3-8$

5 Kumar R, Mathur A, Kumar A, Sharma S, Chakraborty S, Chaturvedi UC. Clinical features \& prognostic indicators of Japanese encephalitis in children in Lucknow (India) Indian J Med Res 1990;91:321-327

6 Yun SI, Lee YM. Japanese encephalitis: the virus and vaccines. Hum Vaccin Immunother 2014;10(2):263-279

7 Kumar S, Misra UK, Kalita J, Salwani V, Gupta RK, Gujral R. MRI in Japanese encephalitis. Neuroradiology 1997;39(3):180-184

8 Dung NM, Turtle L, Chong WK, et al. An evaluation of the usefulness of neuroimaging for the diagnosis of Japanese encephalitis. J Neurol 2009;256(12):2052-2060

9 Hegde AN, Mohan S, Lath N, Lim CCT. Differential diagnosis for bilateral abnormalities of the basal ganglia and thalamus. Radiographics 2011;31(1):5-30

10 Misra UK, Kalita J. A comparative study of Japanese and herpes simplex encephalitides. Electromyogr Clin Neurophysiol 1998;38(1):41-46

11 Oyanguren B, Sánchez V, González FJ, et al. Limbic encephalitis: a clinical-radiological comparison between herpetic and autoimmune etiologies. Eur J Neurol 2013;20(12):1566-1570

12 Saha M, Kumar S, Das A, Gupta RK. Similarities and differences of MR findings between Japanese encephalitis and Wilson's disease. Eur Radiol 2002;12(4):872-876

13 Bousser MG, Ferro JM. Cerebral venous thrombosis: an update. Lancet Neurol 2007;6(2):162-170

14 Kashyap AS, Anand KP, Kashyap S. Thrombosis of the cerebral veins and sinuses. N Engl J Med 2005;353(3):314-315

15 Ameridou I, Spilioti M, Amoiridis G. Bithalamic infarcts: embolism of the top of basilar artery or deep cerebral venous thrombosis? Clin Neurol Neurosurg 2004;106(4):345-347

16 Zeidler M, Sellar RJ, Collie DA, et al. The pulvinar sign on magnetic resonance imaging in variant Creutzfeldt-Jakob disease. Lancet 2000;355(9213):1412-1418

17 Tschampa HJ, Mürtz P, Flacke S, Paus S, Schild HH, Urbach $\mathrm{H}$. Thalamic involvement in sporadic Creutzfeldt-Jakob disease: a diffusion-weighted MR imaging study. AJNR Am J Neuroradiol 2003;24(5):908-915

18 Basumatary LJ, Raja D, Bhuyan D, Das M, Goswami M, Kayal AK. Clinical and radiological spectrum of Japanese encephalitis. J Neurol Sci 2013;325(1-2):15-21
19 Verma R. MRI features of Japanese encephalitis. BMJ Case Rep 2012;2012:bcr0320126088

20 Shoji $\mathrm{H}$, Kida $\mathrm{H}$, Hino $\mathrm{H}$, et al. Magnetic resonance imaging findings in Japanese encephalitis. White matter lesions. J Neuroimaging 1994;4(4):206-211

21 Abe T, Kojima K, Shoji H, et al. Japanese encephalitis. J Magn Reson Imaging 1998;8(4):755-761

22 Misra UK, Kalita J, Phadke RV, et al. Usefulness of various MRI sequences in the diagnosis of viral encephalitis. Acta Trop 2010;116(3):206-211

23 Baringer JR. Herpes simplex virus encephalitis. In:Davis LE, Kennedy PGE. eds.Infectious Diseases of the Nervous System. Oxford Butterworth-Heinemann; 2000:139-164

24 Prakash M, Kumar S, Gupta RK. Diffusion-weighted MR imaging in Japanese encephalitis. J Comput Assist Tomogr 2004; 28(6):756-761

25 Arahata Y, Fujii K, Nishimura T, Uchida T, Kitazawa K, Honda A. Longitudinal magnetic resonance imaging changes in Japanese encephalitis. Brain Dev 2019;41(8):731-734

26 Keng LT, Chang LY. Japanese encephalitis. CMAJ 2018; 190(21):E657

27 Sener RN. Diffusion MRI in Rasmussen's encephalitis, herpes simplex encephalitis, and bacterial meningoencephalitis. Comput Med Imaging Graph 2002;26(5):327-332

28 Handique SK, Das RR, Barman K, et al. Temporal lobe involvement in Japanese encephalitis: problems in differential diagnosis. AJNR Am J Neuroradiol 2006;27(5):1027-1031

29 Borah P, Dhingani DD, Gogoi RK. MR imaging spectrum of Japanese B Encephalitis in the pediatric age group. J Evol Med Dental Sci 2015;4(93):15863-15867

30 Agarwal SL, Ghosh M, Afroze S, Palit A, Ghosh A, Nayek K. Neuroimaging evaluation of pattern of brain involvement in Japanese encephalitis and other viral encephalitis in paediatric age group. Int J Biomed Res 2018;09(4):132-136

31 Kalita J, Misra UK, Pandey S, Dhole TN. A comparison of clinical and radiological findings in adults and children with Japanese encephalitis. Arch Neurol 2003;60(12):1760-1764

32 Handique SK, Das RR, Saharia B, Das P, Buragohain R, Saikia P. Coinfection of Japanese encephalitis with neurocysticercosis: an imaging study. Am J Neuroradiol 2008;29(1):170-175

33 Singh P, Kalra N, Ratho RK, Shankar S, Khandelwal N, Suri S. Coexistent neurocysticercosis and Japanese B encephalitis: MR imaging correlation. AJNR Am J Neuroradiol 2001;22(6):1131-1136

34 Sivamani K, Dhir V, Singh S, Sharma A. Diagnostic dilemmadengue or Japanese encephalitis? Neurol India 2017; 65(1):105-107

35 Md Noh MSF, Rashid AM, Zaidi WA, Khoo CS, Rajadurai N, Muda AS. Neuroimaging in dengue: CT and MRI features. Neurol Clin Neurosci 2018. Doi: 10.1111/ncn3.12231

36 Jia M, Xiong N, Huang J, et al. Japanese encephalitis accompanied by cerebral venous sinus thrombosis: a case report. BMC Neurol 2012;12:43

37 Neo RJ. Japanese Encephalitis presenting with cerebral venous sinus thrombosis: a case report. Med J Malaysia 2019; 74(6):537-539

38 Mokkappan S, Basheer A, Iqbal N, Chidambaram S. Bilateral thalamic bleed and cerebral venous sinus thrombosis in Japanese encephalitis. BMJ Case Rep 2015;2015:bcr2014207957 